\title{
Exercise for people with hip or knee osteoarthritis: a comparison of land-based and aquatic interventions
}

This article was published in the following Dove Press journal:

Open Access Journal of Sports Medicine

22 July 2010

Number of times this article has been viewed

\author{
Ann E Rahmann \\ Division of Physiotherapy, School of \\ Health and Rehabilitation Sciences, \\ The University of Queensland, \\ St Lucia, Queensland, Australia
}

\begin{abstract}
Expert opinion considers the referral of people with osteoarthritis (OA) for physiotherapy to be a core component of managing the functional disability and pain of the disease. Clinical guidelines for the physiotherapy management of people with OA focus on three main areas: exercise, pain relief, and specific manual therapy techniques. Land-based group and individual physiotherapy exercise programs, as well as manual therapy, have demonstrated a distinct benefit in favor of physiotherapy intervention. Similarly, both general and specific aquatic physiotherapy exercise programs have shown positive outcomes for people with OA. This review will focus primarily on therapeutic exercise to improve strength and fitness and reduce pain in people with hip or knee OA. An overview of the principles of hydrodynamics relevant to aquatic exercise is also included to facilitate an understanding of effective aquatic exercise programs. The issue of compliance with exercise programs will also be discussed. Clinicians will, therefore, gain an understanding of the benefits of land-based and aquatic exercise for people with OA.
\end{abstract}

Keywords: exercise, physical therapy, aquatic therapy, hip and knee osteoarthritis, strength, pain, aerobic exercise

\section{Introduction}

Historically, osteoarthritis (OA) was considered a simple consequence of aging, but current opinion contends that while aging of the musculoskeletal system predisposes a person to the development of arthritis, it does not actually cause OA. ${ }^{1}$ Instead the disease is multifactorial and results from a combination of external factors, including muscle weakness and reduced proprioception, and changes within the joint itself, such as inflammation, subchondral and trabecular bony remodeling, and usually cartilage erosion. ${ }^{1,2}$ Preexisting factors, such as obesity and previous joint injury, also increase the likelihood of developing OA. ${ }^{3}$ In the later stages of the disease, pain and inefficient muscle control around the joint in combination with ligamentous laxity and joint instability or stiffness can significantly affect functional ability. ${ }^{4}$ Although the disease process in both hip and knee arthritis is progressive, hip OA tends to deteriorate more quickly than knee OA. ${ }^{5}$ Elective joint replacement surgery is usually the only option at the later stage of the disease process when conservative management is no longer effective. ${ }^{6}$

Core components of management in the early stages of OA include pharmacotherapy, education, weight loss, and physiotherapy. ${ }^{7,8}$ Recommended exercise programs for people with OA include strengthening, flexibility, and aerobic fitness. ${ }^{9,10}$ High-quality research evidence has demonstrated that strengthening exercises are particularly effective to improve function and reduce pain in OA. ${ }^{8}$ Interventions targeted at improving strength and function in the late stages of OA, before surgery, can still be beneficial ${ }^{11}$ 
because people with poorer preoperative function do not attain the same functional level after surgery as those with less preoperative disability. ${ }^{12}$ Aquatic exercise is commonly recommended for people with $\mathrm{OA},{ }^{13}$ particularly in the later stages of the disease process when land-based activities are more difficult. ${ }^{9}$ Evidence, therefore, exists of the benefit of exercise intervention in both the early and late stages of the disease. Clearly, with the complexity of factors involved at every stage of the osteoarthritic disease process, there is no single treatment that can improve strength and function, reduce pain, and prevent disease progression. ${ }^{14}$ Optimal management for each individual is, therefore, dependent on the stage of the disease process and the ability of health professionals to select interventions appropriate for that individual at that time.

The particular focus of this review is therapeutic aquatic exercise for people with OA. Hydrotherapy is a broad, general term for the remedial use of water. In addition to indicating therapeutic exercise in water, hydrotherapy can include immersion in mineralized water (balneotherapy) or turbulent spa therapy, ${ }^{15}$ and Kniepp hydrotherapy, which involves pouring water over the affected limb in standing ${ }^{16}$ and alternating hot- or cold-bath immersion of a limb. ${ }^{17}$ Because only English-language publications could be considered, much of the European hydrotherapy research-base could not be included in this review. However, a systematic review, including a large number of non-English language RCTs, reported that balneotherapy in rheumatology improved pain and quality of life. ${ }^{18}$ A more recent Cochrane review of the small number of English-language studies of balneotherapy came to a similar conclusion but cautioned that further large well-designed trials were needed. ${ }^{15}$ People with OA may find one or all of these different water-based activities helpful at various stages of the disease process, although the availability of specific types of aquatic activities often depends on geographical locality. For example, spa therapy is very common across Europe and North America, although frequently at expensive spa resorts, ${ }^{9}$ but is not often available elsewhere.

Over the last 5-10 years, the terms aquatic physiotherapy and aquatic therapy have been used in published literature and by professional associations, such as the World Confederation for Physical Therapy, ${ }^{19}$ The Chartered Society of Physiotherapy in the United Kingdom, the American Physical Therapy Association, and the Australian Physiotherapy Association, to differentiate physiotherapy-specific interventions from more general hydrotherapy practices. Rather than using the generic term of hydrotherapy, this review will follow the recently established convention and use aquatic physiotherapy to indicate intervention that is physiotherapyspecific and aquatic exercise as a general term for therapeutic exercise in water. Treatment methods, such as balneotherapy and spa therapy will therefore, not be discussed.

\section{Why is improving strength important in OA?}

Changes in muscle activation, strength, and somatosensory function occur with normal aging. ${ }^{20,21}$ Maintenance of adequate muscle strength is crucial when aging ${ }^{22}$ and has been strongly linked to functional ability. ${ }^{23}$ In older people with unilateral hip OA, marked differences in strength, muscle mass, activation, and force generation have been demonstrated in all the major hip and knee muscles of the arthritic limb when compared with the unaffected limb. ${ }^{24,25}$ Similar changes have been found in people with knee OA, ${ }^{26}$ with some authors contending that strength deficits of the quadriceps may be a precursor to the development of knee OA and not simply a consequence of reduced activity levels. ${ }^{27,28}$ Muscle activation controls loading through the knee and hip during gait in healthy adults, ${ }^{29}$ and decreased loading and alterations in biomechanical forces related to changed muscle activation have been implicated in the progression of arthritic disease. ${ }^{30,31}$ Increased overall activation time and cocontraction of muscles around the knee compared with healthy controls were demonstrated in people with knee OA and was thought to result from an attempt to stabilize the degenerating joint during weight bearing. ${ }^{32}$ People with severe knee OA also had stronger hip adductors than those with mild OA which, because of the direction of muscle force, may act to compensate for the increasing varus deformity of the knee. ${ }^{33}$ Although this increased adductor activation seems the opposite of what is needed, others consider that it may simply reflect the only mechanism available to achieve a degree of stability. ${ }^{34}$ Far fewer studies have investigated people with hip OA, but motor control dysfunction in the ipsilateral gluteus medius during a stepping task has been identified. ${ }^{35}$ This alteration in hip muscle function could be a precursor or a consequence of hip OA. ${ }^{36}$ Reduced hip abductor strength has also been shown in people with knee pathology ${ }^{37}$ and is most likely to be a consequence of altered loading during gait to rapidly move body weight onto the unaffected $\operatorname{limb} .{ }^{38}$ In contrast, medial knee OA progressed more slowly in people with stronger ipsilateral hip abductors ${ }^{39}$ because adequate hip abductor strength may control weight shift and maintain lateral pelvic stability during the single-leg stance phase of gait. $^{40,41}$ Despite this evidence of more widespread muscle weakness in knee OA, exercise programs have traditionally 
focused primarily on quadriceps strengthening. ${ }^{42}$ Few studies have investigated exercise programs specifically for hip OA. Clinicians must remember that muscle atrophy within the arthritic limb is widespread ${ }^{25,43}$ and exercise interventions for OA need to consider the biomechanics of the whole lower limb and trunk. ${ }^{38}$

\section{Land-based strengthening exercise in OA}

The aim of strengthening exercises in people with OA is primarily to improve control and stability of the joint during movement and thus maintain functional ability. A metaanalysis of 22 research studies determined that an effective strengthening exercise program for OA needed to incorporate both joint-specific and more general exercises, with progression of the exercises and compliance also being important factors. ${ }^{44}$ More recent reviews also indicated a strong evidence base for the efficacy of strengthening exercises in managing OA. ${ }^{2,45}$ However, the common conclusion of systematic reviews over the last 10 years is that although exercise has been shown to be extremely beneficial, there is, as yet, insufficient evidence to recommend the optimal type of exercise, intensity, or dosage for people with hip or knee OA. ${ }^{46-48}$

Conversely, a recent systematic review determined that quadriceps-strengthening exercises in markedly malaligned or unstable knees might in fact be detrimental because of the alteration in loading through the joint during quadriceps contraction. ${ }^{49}$ Other researchers have also suggested that subgroups of people with knee OA, such as those with patello-femoral OA, may require specific exercise programs for optimal efficacy ${ }^{50}$ and an RCT is currently being undertaken to determine if this is actually the case..$^{51}$ In contrast to knee OA, very little research has been undertaken that has specifically investigated exercise for hip OA. ${ }^{48}$ This paucity of research may be due to the complexity of muscle activity and biomechanics around the hip in comparison to the knee and because pelvic stability during function involves bilateral components. ${ }^{36}$ Clinicians have suggested that, just as with knee OA, subgroups of the hip arthritis population may require a different intervention focus. ${ }^{52}$ An exercise approach more targeted to disease stage and specific functional deficits may provide better overall outcomes ${ }^{53}$ and further research is clearly needed to inform clinical practice.

\section{Why aquatic exercise is different to exercise on land}

Understanding the basic principles of hydrodynamics allows the development of effective aquatic exercise programs and is an important basis on which to examine the differences between land and aquatic exercises. When considering strengthening exercise in the water, the amount of drag or turbulence and thus resistance created by a movement is related to the speed and shape of the object moving through the water. ${ }^{54}$ The size and shape of the moving body part, the speed of the activity, and the depth of water in which the movement occurs determine the effort and intensity of an exercise. ${ }^{54}$ For example, doubling the speed of a movement in water increases the drag and, therefore, resistance four-fold. ${ }^{55}$ Exercising in water at the same speed as on land is significantly harder because of the turbulence created and the viscosity of water ${ }^{56,57}$ Viscosity also allows a safety margin in aquatic exercise because if pain is experienced during an exercise, as soon as the limb stops moving, the resistance immediately ceases and buoyancy assists the support of the limb. ${ }^{54}$ This allows aquatic exercise to be completed within the limits of pain and under the control of the person exercising. ${ }^{58}$ Resistance in the water can be increased by adding equipment, such as paddles or flippers. ${ }^{59,60}$ However, if specific details of depth, speed, and equipment used are not included in published reports, it becomes very difficult to compare results between trials and for clinicians to replicate successful protocols. ${ }^{61}$ Attempts have been made to quantify the resistance during aquatic exercise ${ }^{62,63}$ and its relationship to muscle activity, ${ }^{64,65}$ but it remains a difficult task in everyday clinical practice because calculating the forces related to movement in water is extremely complex. ${ }^{66}$ Thus, it is more difficult to precisely calibrate the progressions of aquatic resistance exercise in comparison to land-based exercise. An additional factor that must also be considered is that functional activities, such as step-ups on land and in water, appear similar but are not the same in terms of load, force, or biomechanics. Considering an average person of $80 \mathrm{~kg}$, stepping up a $15-\mathrm{cm}$ step on land requires lifting the entire load of $80 \mathrm{~kg}$. In contrast, when stepping up onto a similar height step in water, the load is dependent on the depth. For example, in waist-deep water ( $50 \%$ weight-bearing), the load may only be around $40 \mathrm{~kg}$ (ie, $50 \%$ of the total load). Whether muscle activation patterns are similar, despite the reduced load in water is not yet known, and further research is required to guide clinical decision making.

Two additional principles of hydrodynamics also make aquatic exercise different to land-based exercise and are important to consider clinically. The first is hydrostatic pressure, which increases with water depth, $1 \mathrm{mmHg}$ every $1.36 \mathrm{~cm}$, so when immersed at $1.2 \mathrm{~m}$, the resulting pressure around the limb is higher than diastolic pressure and thus 
aids venous return. ${ }^{54}$ This principle underpins the clinical finding of reduced pregnancy-related edema and lymphedema after immersion. ${ }^{67,68}$ Another small RCT found that swelling around the knee early after joint replacement surgery was $3.7 \mathrm{~cm}$ less ( $95 \%$ confidence interval: -7.1 to $-0.3 \mathrm{~cm}$ ) in those who had completed aquatic physiotherapy while in the hospital compared with the control group, who only completed ward-based exercise. ${ }^{69}$ Guidelines for the management of $\mathrm{OA}$ recommend water exercise to improve range of movement, ${ }^{14}$ and the reduction in edema from the effect of hydrostatic pressure during immersion is likely be a contributing factor.

The second principle involves thermodynamics. Water retains its temperature effectively because of its high specific heat and is also an efficient heat conductor so that warmer water readily transfers heat to the immersed body. ${ }^{70} \mathrm{~A}$ wide range of water temperatures are used clinically from cold temperatures (around $15^{\circ} \mathrm{C}$ ) to reduce delayed onset muscle soreness and hasten recovery after strenuous exercise ${ }^{71}$ and cooler temperatures $\left(25^{\circ} \mathrm{C}-29^{\circ} \mathrm{C}\right)$ for conditioning exercise. ${ }^{72}$ Therapeutic exercise is usually undertaken in thermoneutral water $\left(33.5^{\circ} \mathrm{C}-35.5^{\circ} \mathrm{C}\right)$ because the physiological effects of immersion are minimized, ${ }^{73}$ allowing sufficient immersion time to complete an exercise program. ${ }^{74}$ Guidelines for the management of OA recommend heat and cold therapies as low-risk and low-cost nonpharmacological options, ${ }^{7}$ although evidence of their efficacy in OA is not as strong as that for other interventions like strengthening exercises. ${ }^{75}$ Aquatic exercise in thermoneutral water allows exercises to be undertaken, while the affected limb remains warm and has circumferential compression applied by hydrostatic pressure, a combination that is not possible to replicate on land. Whether the hydrodynamics of aquatic exercise translates into greater efficacy compared with land-based exercise for people with $\mathrm{OA}$ requires consideration.

\section{Aquatic exercise to improve strength and function}

A thorough systematic review of the overall evidence base for hydrotherapy published in 2002 concluded that the small number of moderate- to high-quality trials indicated a beneficial effect of aquatic exercise on strength, function, and self-efficacy in rheumatic diseases, such as OA. ${ }^{61} \mathrm{~A}$ more recent Cochrane systematic review of aquatic exercise for knee and hip arthritis similarly concluded that aquatic exercise had a positive effect on function and pain in the short term and could be used as the initial component of a longer term intervention for people with arthritis. ${ }^{76}$ To decide whether aquatic exercise can improve strength and function in $\mathrm{OA}$, it is important to first consider whether strength can be increased by aquatic exercise. Then, by comparing aquatic exercise to other interventions, it should be possible to determine if aquatic exercise offers any additional benefit over land-based exercise.

Poyhonen and colleagues ${ }^{55}$ randomized a group of healthy young women into either a 10 -week progressive, aquatic resistance training protocol or a nonintervention control group and found both isometric and dynamic torque of the quadriceps and hamstrings improved in the aquatic training group by week 10. Isometric force in the aquatic group was the only variable to improve between the baseline and the week 5 measurements. The greatest improvements in torque values, force-time curves, and lean muscle cross-sectional area in both groups occurred between weeks 5 and 10 rather from baseline to week 5 . The researchers propose this difference occurred because a smaller resistance boot was used in the early weeks, allowing participants in the aquatic group to achieve faster velocities and perhaps facilitating isometric muscle activation. In the later stages, the progression to larger boots created greater drag forces and, therefore, stimulated improved dynamic strength. Another study ${ }^{77}$ compared one specific quadriceps strengthening exercise on land and in water, also using the principle of progressive overload and young women as subjects. The land-based strengthening exercise chosen was knee extension in sitting, where the load was progressed using sandbag weights. A comparable starting position for the aquatic exercise was achieved with participants lying prone at the pool edge, with both legs in the water and the dominant knee flexed to $90^{\circ}$. Resistance was created by pushing down against the buoyant force of empty 2-L bottles attached to the lower leg. Increasing the number of bottles used increased the buoyant force and, therefore, resistance. After 7 weeks, both protocols achieved similar, significant improvement in isotonic strength. These studies both demonstrate that aquatic exercise can achieve similar gains in lower limb muscle strength to land-based protocols, but a thorough understanding of hydrodynamics is necessary to ensure the aquatic exercises are designed with a comparable training load.

Specific aquatic exercises can, therefore, improve strength in a healthy young population, but what is the effect of aquatic exercise in the older arthritic population? Generic aquatic exercise classes, such as the Arthritis Foundation Aquatic Program (AFAP), that are run in community facilities by trained instructors have been compared with nonintervention control groups. ${ }^{78-81}$ The intervention period in three of 
the trials lasted between 6 and 12 weeks with no long-term follow-up. Two trials incorporated a 12-month community intervention with one trial having no further follow-up ${ }^{82}$ and the second trial including reassessment 6 months after completing the exercise program. ${ }^{81}$ The aquatic exercise group in all the trials generally showed some improvements in strength, flexibility, and functional ability compared with the control groups. The exception was the RCT with the 12-month intervention period, which showed no change in strength at any time. The results of this trial may have been influenced by the fact that less than $60 \%$ of participants attended classes at the recommended frequency and the authors commented that the exercise program itself might have been too gentle to improve strength. A more recent RCT compared a twice-weekly, specific aquatic physiotherapy program run for 6 weeks in small groups by an experienced aquatic physiotherapist with a nonintervention control group. ${ }^{83}$ Significant improvements in pain, function, and hip abductor strength were found in the aquatic group after the intervention. The exercise program in this trial may have been more effective than the general exercise classes because it was based on hydrodynamic principles and included functional strengthening exercises, such as squats and step-ups. Evidence, therefore, supports therapeutic aquatic exercise, whether delivered in a general class or as a specific physiotherapy intervention, to improve strength and function more than no intervention at all.

As discussed previously, there is strong evidence supporting the use of land-based exercise for strengthening in people with OA ${ }^{84}$ It is, therefore, important to also compare aquatic exercise to land-based exercise, rather than just a control group that undertakes no comparable intervention, to determine whether aquatic exercise offers any additional benefit over land-based strengthening protocols. A recent Cochrane review identified only six trials that compared aquatic- and land-based interventions in the treatment of hip or knee OA and concluded that, although there appeared to be a shortterm benefit, the small number of RCTs limited further recommendations. ${ }^{76}$ Several trials have been published since this review in 2007. The effect of therapeutic exercise for hip OA was analyzed in a meta-analysis that included trials utilizing aquatic exercise. ${ }^{85}$ Despite finding that strengthening exercises were effective for hip OA, trial results were pooled, with land and aquatic programs not specifically compared. Four trials comparing the effect of aquatic- and land-based exercises found little difference in outcomes. ${ }^{86-89}$ Two other trials found that a gym-based class achieved better strength gains than an aquatic exercise program $;{ }^{74,90}$ however, the method of statistical analysis used in one trial is complex and unclear, so interpreting the results is difficult. ${ }^{76}$ Several of the more recent trials explicitly state that the aim was to compare the same exercises on land and in water. ${ }^{74,87,88}$ Earlier discussion in this review has emphasized that exercise in water is different to land because of hydrodynamics and that consideration of these principles is needed to design an effective aquatic program. It is possible that efforts to standardize intervention protocols in these research studies may have influenced the results because the aquatic strengthening component was not optimal. In fact, Foley et $\mathrm{al}^{90}$ commented that the content of the aquatic program that they used may explain why the aquatic group showed greater improvements in aerobic fitness than strength. A recent RCT compared a specific aquatic exercise program to land-based TaiChi classes for people with knee OA. ${ }^{91}$ Although both interventions showed improvements in pain and self-reported function lasting up to 12 weeks after completing the program, objective physical performance measures showed greater improvement in the aquatic exercise group, indicating that a specific aquatic program may offer advantages over generic land-based exercise.

Clinicians often recommend aquatic exercise for people with OA to improve their strength and function. ${ }^{13,14}$ Research to date has demonstrated that aquatic exercise is more effective at improving strength and function when compared with no intervention, but whether a physiotherapist-supervised or designed program is any more effective than a generic aquatic exercise program has yet to be determined. When comparing aquatic exercise to land-based exercise, it would appear from the few published studies that aquatic exercise is less effective than similar land-based protocols to improve strength. However, most studies till date have not used aquatic exercise programs based on the principles of hydrodynamics to maximize the potential for strengthening. Further studies need to consider the aquatic exercise component more carefully but, just as with land-based exercise, optimal aquatic exercise program content is not yet clear.

\section{Why is managing pain important in OA?}

Pain is one of the most common symptoms reported by people with OA, with the control and effective management of pain considered one of the key goals of intervention in OA. ${ }^{14,92}$ Poorly controlled pain is a primary reason for progressing to joint replacement surgery. An individual's perception of pain severity can also be influenced by centrally mediated factors and behavioral components ${ }^{93}$ because the experience of pain is 
a complex and dynamic process..$^{94,95} \mathrm{OA}$ pain can result from local factors, such as synovial inflammation or subchondral bony destruction. ${ }^{96}$ Pain can inhibit muscle activation in OA and, therefore, contribute to the loss of strength, alteration in loading, and changes in gait velocity. ${ }^{97-99}$ People with OA also demonstrate worse static and dynamic balance ${ }^{100}$ and use different strategies to negotiate obstacles than healthy elders. ${ }^{101}$ Pain-relieving intra-articular injections improved single limb balance and reduced tripping over obstacles in people with knee OA, but the level of improved ability did not reach that of healthy age-matched controls. ${ }^{102}$ In contrast, a previous study ${ }^{103}$ demonstrated that pain relief by joint injection improved quadriceps maximum voluntary contraction in arthritic knees but made no difference to proprioception and postural sway in standing. The authors acknowledge that static balance requires efficient control of more muscles than just the quadriceps and a period of exercise intervention may be required to retrain overall stability in standing. Other authors have expressed the view that pain has a protective role as a mechanism to control loading and that reducing pain without improving strength and control around the joint may hasten degeneration. ${ }^{104}$ Exercise has, therefore, been widely promoted in recent years as a way of managing pain in OA, but why it is effective is less clear. Strengthening the muscles surrounding the arthritic joint is thought to assist in reducing pain by improving the control of the arthritic joint during movement, ${ }^{45,105,106}$ but pain inhibition is also considered to play a role in the development of weakness. ${ }^{97}$ Participants in exercise interventions for $\mathrm{OA}$ also have reported improved overall well-being, even when objective performance measures have not significantly changed ${ }^{107}$ and so the effect of exercise on pain in $\mathrm{OA}$ is multifactorial. ${ }^{44}$

Clearly, the interplay between pain, muscle contraction, and functional ability in people with OA is complex. Self-reported outcome measures are frequently used in trials to measure the impact of interventions on both pain and functional ability. The Western Ontario and McMaster Universities Index (WOMAC) is one of the most commonly used worldwide and includes both pain and functional subscales. ${ }^{108}$ Recent research has found that self-reported measures of function, such as the WOMAC scale, can be strongly influenced by the individual's perception of pain and effort during daily activities and is, therefore, not necessarily a true measure of the individual's actual ability at that point in time. ${ }^{109}$ When reviewing trials reporting the effect of an intervention on pain and function in OA, it is important to determine whether self-report or performancebased measures of functional ability, such as the Timed
Up and Go (TUG) test ${ }^{110}$ or a timed walk, ${ }^{111}$ were used. The combination of both self-report and performancebased measures has been recommended, as it is likely to offer a more complete picture of the efficacy of different interventions. ${ }^{12}$

\section{Land-based exercise and manual therapy for pain management}

Medication is the primary method of managing arthritic pain recommended in published guidelines. ${ }^{7113}$ However, an overview of systematic reviews focusing on physiotherapy for knee OA reported high-quality evidence supporting the use of exercise to reduce pain and improve function. ${ }^{114}$ Another recent systematic review identified 18 high-quality trials and concluded that resistance training improved selfreported pain and physical function in $50 \%-75 \%$ of the combined cohort. ${ }^{115} \mathrm{~A}$ further comprehensive meta-analysis of 21 trials also concluded that strengthening exercises alone can reduce pain in people with OA, but optimal intervention also includes functional training and aerobic exercise. ${ }^{44}$ The majority of published systematic reviews and meta-analyses focus on interventions for knee OA. The primary evidence for the efficacy of exercise in hip OA is expert opinion rather than clinical trials. An umbrella review published in 2007 specifically sought trials reporting interventions for hip OA. ${ }^{116}$ In contrast to the effect of exercise in knee OA, this overview found only low-quality evidence for the benefit of exercise in hip OA. As the authors pointed out, this is due to both the small number of trials involving hip OA and also because many reviews and research trials combine knee and hip OA so it is difficult to determine whether hip OA responds differently to exercise. Another review concluded that strengthening exercise had a beneficial effect on pain in people with hip $\mathrm{OA}^{85}$ but further research involving people with hip OA is clearly needed. ${ }^{48}$ Although evidence supports the efficacy of exercise in reducing the pain associated with OA, the optimal exercise regime and long-term effect have not yet been clarified. ${ }^{47,17}$ One small study highlighted that regular, ongoing exercise maintained a reduction in pain beyond 12 months from initial assessment but clearly further studies are needed. ${ }^{118}$

\section{Manual therapy for OA}

Manual therapy is frequently used clinically in combination with exercise therapy for people with OA and commonly includes active and passive joint mobilizations, stretching, and soft tissue massage with overall aim of reducing pain, normalizing tissue and joint biomechanics, and improv- 
ing function. ${ }^{31}$ Specific techniques vary widely amongst practitioners and from region to region across the world, and it is beyond the scope of this review to discuss manual therapy techniques in detail. The combination of manual therapy and exercise has demonstrated superior functional improvements in people with OA when compared with either a placebo control ${ }^{119}$ or an independent home exercise program, ${ }^{120}$ but whether it offers any additional benefits over a land-based strengthening program has not yet been investigated.

\section{Aquatic physiotherapy for pain management}

A recent meta-analysis of trials investigating water-based exercise, aerobic and strengthening exercises, and spa therapy for OA concluded that all have a positive effect on pain. ${ }^{121}$ A Cochrane review of aquatic therapy for OA of the hip or knee also concluded that pain may be decreased by aquatic therapy.$^{76}$ Because of the water temperature and the decreased loading, aquatic exercise is often considered an ideal place to begin exercise or for those in the more advanced stages of the disease where exercise on land has become too difficult. ${ }^{76}$ Clinicians often contend that aquatic physiotherapy offers greater pain relief than land-based intervention in musculoskeletal conditions, but a recent systematic review failed to find sufficient research evidence to support this suggestion. ${ }^{122}$ One study comparing similar land- and water-based exercises for people with knee OA showed no significant differences between the interventions in range or walking distance but postexercise pain was less in the water-exercise group. ${ }^{87}$ Similarly, when comparing aquatic training group and land-based progressive resistance training group, the aquatic training group reported significantly less postexercise pain. ${ }^{77}$ People with late-stage OA awaiting joint replacement surgery experienced less pain immediately after an aquatic exercise program than those who undertook land-based exercise. ${ }^{89}$ Orthopedic inpatients completing an intensive aquatic exercise program also reported significantly less pain during intensive aquatic exercise and perceived less difficulty than when undertaking usual ward exercise. ${ }^{123}$ However, aquatic exercise for people in pain must be undertaken with caution clinically because reduced pain perception during aquatic exercise may make it easier to over-exercise because the perceived workload is less than during landbased exercise. Although systematic reviews were unable to confirm the clinical contention that aquatic exercise can reduce pain, the methodological processes required in metaanalysis may dilute the results of the few, small positive trials.
Further research is clearly warranted to determine whether this clinical perception is supported.

Aquatic exercise may have an effect on pain because of buoyancy and the hydrodynamics related to moving in water. ${ }^{124}$ The effect of buoyancy could reduce pain during exercise because the depth of immersion is directly related to the percentage weight bearing. ${ }^{70}$ Weight bearing when immersed to the neck is considered to be $10 \%, 30 \%$ at the xiphisternum, and $50 \%$ at the waist. ${ }^{125}$ To reduce load and pain in the joints, the individual can exercise in deeper water or conversely to progress weight bearing and load, exercises can be undertaken in shallower water. ${ }^{126}$ The ability to easily alter weight bearing to reduce pain during functional exercises is not possible in land-based exercise programs. A small clinical trial recently demonstrated that aquatic immersion restored normal spinal stature more effectively than an equivalent land-based position, adding support for the argument that immersion decreases joint loading. ${ }^{127}$ Immersion in thermoneutral temperature water is commonly thought to reduce pain because sympathetic nervous system conduction slows, thus reducing pain perception. ${ }^{70,128}$ The compressive effects of hydrostatic pressure in combination with the circulatory changes that occur with immersion reduce swelling, allowing greater movement to reduce joint and soft-tissue stiffness and, therefore, improve pain levels. ${ }^{79,82,83}$ To date, one small $\mathrm{RCT}^{69}$ did show a difference between a general water exercise program and a specific aquatic exercise program designed with an understanding of hydrodynamics and movement in water. Whether the positive effect of aquatic exercise on pain found clinically is due simply to the hydrodynamics of immersion or is related instead to the aquatic exercise intervention requires further research.

Just as in a land-based physiotherapy practice, in aquatic physiotherapy practices where the physiotherapists are in the water and able to treat people individually, it is common clinical practice to include specific manual therapy techniques with the aquatic exercise program. ${ }^{126,129}$ No research is available as yet to guide clinical decision making in this area of practice, but the rationale is the same as for land-based manual therapy and is worthy of further investigation.

\section{Why is aerobic exercise important in OA?}

People with OA are often deconditioned compared with their peers and, because of the arthritis, are often unable to exercise at sufficient pace to improve or maintain cardiovascular fitness. ${ }^{130}$ Obesity is an independent risk factor for developing $\mathrm{OA}^{95}$ and weight loss is recommended because 
it can reduce disease severity and progression. ${ }^{14,113,131}$ In the later stages of the disease, it is frequently difficult to exercise at sufficient intensity on land to achieve aerobic training so nonweight bearing exercises, like cycling, water walking, and swimming, are often recommended at this point. ${ }^{113}$ Results of a systematic review supported the recommendation that aerobic exercise has both shortand long-term benefits for people with OA, with possible options, including walking, running, cycling, and aquatic exercise. ${ }^{130} \mathrm{~A}$ more recent meta-analysis also concluded that aerobic exercise offered significant benefits for the OA population. ${ }^{132}$ Interestingly, when comparing strengthening and aerobic exercise, strengthening seems to offer better short-term benefits in terms of impairments, such as pain, but longer-term functional gains seem to be enhanced by aerobic exercise. ${ }^{45}$ To determine whether high- or lowintensity exercise was any more effective in the arthritic population, a recent Cochrane review was completed. ${ }^{133}$ Only one trial comparing the two levels of intensity was identified, and it found both protocols were an equally effective method of improving function and aerobic capacity in those with knee OA. ${ }^{134}$ Further research is clearly needed to determine optimal program content.

\section{Aquatic exercise for aerobic fitness}

Just as with research into strengthening exercise, the majority of trials to date have evaluated aerobic exercises against a nonactive control group. One of the few trials to compare active interventions randomized participants with OA or rheumatoid arthritis (RA) into a 12-week walking, aquatic aerobic exercise or range of motion exercise control group. ${ }^{135}$ Significant improvements in endurance, self-reported disability, and objective performance measures were demonstrated, even up to a year after baseline assessment in both the walking and aquatic exercise groups. Two important aspects of this study need to be considered. First, results of those with OA and RA were not analyzed separately so it is not possible to determine the effect solely for those with OA. Second, the authors report that over $60 \%$ of participants continued to exercise on average 2 hours per week at the 1-year measurement period, the most likely explanation for the maintenance of functional improvement at this time point. This continuation of exercise is unusual as most reviews report significant drop-off in compliance over the long term in other studies. ${ }^{42,46}$

Walking is the most common aerobic exercise to be investigated and has been shown to be beneficial and, importantly, not to exacerbate arthritic symptoms. ${ }^{136,137}$ Walking in water is also commonly a component of aquatic exercise programs and often used for gait reeducation, as well as for aerobic exercise in people with arthritis. ${ }^{89,90}$ Walking in water is not the same as walking on land because of the turbulence, drag forces, buoyancy, and viscosity as discussed previously. ${ }^{54}$ Both comfortable and maximal walking speed are around 30\% less in water than is possible on dry land. ${ }^{138}$ Older adults ${ }^{139}$ and those with RA $^{140}$ who walked in water and on land at a similar pace reported higher levels of exertion in the water component. Although walking faster in water to facilitate an aerobic training effect is possible, careful consideration of the forces through the lower limbs is needed. When walking as fast as possible in water, maximum ground reaction forces were similar to land-based values, despite the slower maximal walking speed that could be achieved in water. ${ }^{141}$ Walking in chest deep water reduced loading through the knee during stance, but when walking speed increased, the hip extensor moment in late stance was significantly greater. ${ }^{142}$ Thus, walking in chest deep water may be more comfortable for people with knee arthritis because of the reduced loading during stance, but increasing gait speed to incorporate an aerobic component may actually increase the loading at the hip and, therefore, exacerbate an arthritic hip joint. Compressive load through the patello-femoral joint is reduced when walking backwards and thus walking this way in water may also reduce load through the knee. ${ }^{138,143}$ Careful prescription of water walking, specifying depth, direction, and speed, is needed for people with OA.

Deep-water running is an alternative to swimming and water walking for cardiovascular exercise in the OA population. ${ }^{135}$ This form of exercise involves using a flotation device or belt so that the feet do not touch the bottom of the pool and then moving through the water using various combinations of upper and lower limb movements, with the aim of reaching an appropriate aerobic training level. ${ }^{144}$ Deep-water running for fitness training is well tolerated by healthy, older people and appropriate training levels to improve aerobic fitness can be achieved. ${ }^{145}$ Older overweight women reported a similar rate of perceived exertion for treadmill walking and deep-water running at maximal intensity. ${ }^{72}$

Aerobic exercise is an important component to consider in an holistic approach to managing OA, and evidence exists to support both high- and low-intensity training regimes $^{134}$ and the efficacy of land and aquatic aerobic exercises. ${ }^{132}$ However, as with strengthening exercise, optimal program content is not yet clear. Regardless of 
the efficacy shown in research trials, if regular exercise is not continued, the benefits decline over the longer term. ${ }^{42,45,81}$

\section{Compliance with exercise}

Regardless of all the evidence demonstrating the positive effect of exercise for people with OA, compliance with exercise and life-style changes remain difficult to achieve for many people. ${ }^{8}$ Research studies have clearly demonstrated that people who complete exercise programs or attend allocated classes most regularly have better outcomes. ${ }^{45,53}$ Increased self-efficacy, in addition to increased strength, is a protective factor for disease progression in the early stages after diagnosis of OA. ${ }^{114}$ Aquatic exercise is usually considered an enjoyable form of exercise by participants ${ }^{146}$ and perceived as less effort than land-based exercises. ${ }^{123}$ Compliance with aquatic exercise has also been shown to be superior to land-based exercise programs. ${ }^{88}$ Goal setting is an important component in the management of people with chronic musculoskeletal pain. ${ }^{93}$ Successfully achieving exercise goals have been shown to increase aquatic class attendance and, therefore, improve overall outcomes. ${ }^{147}$ Aquatic exercise has been shown to improve health-related quality of life in older women. ${ }^{145}$ If people with OA find exercising in water an enjoyable and less painful activity, it follows that compliance may be enhanced. The pool environment, with numbers of people exercising together, can be a positive environment and may have an effect on compliance and adherence to exercise for people with OA, over and above the exercise program itself. ${ }^{126}$

\section{Clinical implications}

Optimal management of OA is dependent on the stage of the disease and the selection of interventions appropriate for that individual at that time. ${ }^{14}$ Increasing strength is important for people with OA because strength is closely related to functional ability and can assist the control of biomechanical loading through the arthritic joint. There is currently insufficient evidence to recommend optimal type, intensity, or dosage of land-based strengthening exercises. Evidence from published trials supports the efficacy of resistance training programs using free weights or elastic resistance bands, machine-based exercise, and body-weight resisted functional exercises. However, care needs to be taken when prescribing quadriceps strengthening when the knee is malaligned. Aquatic exercise can increase strength, provided the speed of movement and resistance are sufficient and hydrodynamic principles are understood, but as with land-based exercise, optimal aquatic program content is not yet known. General aquatic arthritis exercise classes can be beneficial, provided people attend regularly. Specific functional aquatic exercise programs are more effective than doing no exercise, and several clearly described protocols incorporating functional strengthening have been published. ${ }^{69,83}$ Clinicians can use these protocols as a starting point when developing their own aquatic programs.

Land-based exercise can assist pain management, but why it is effective is not yet known. Most authors consider that stronger muscles optimize functional loading through the joint and affected limb. Clinicians also commonly recommend aquatic exercise to reduce pain, but insufficient research evidence exists at present to support this contention. ${ }^{122}$ However, participants report less postexercise pain after aquatic exercise than after land-based exercise $^{89}$ and this may enhance compliance with aquatic exercise.

Aerobic exercise is important for general fitness and assisting weight loss in people with OA. Options for landbased aerobic exercise include walking, running, and cycling. Aquatic exercise can be safely undertaken at sufficient intensity to improve cardiovascular fitness and possible options include shallow water walking, swimming, and deep-water running.

\section{Overall summary of the evidence}

Evidence from systematic reviews and RCTs supporting the efficacy of aquatic exercise is increasing every year. ${ }^{148}$ However, even with the far greater volume of research into land-based exercise for people with OA, three key questions still remain to be answered for clinicians - what type of exercise is best, for which particular type of OA presentation and how is this intervention best delivered. ${ }^{53}$ When considering aquatic exercise, there is an additional important question that remains to be answered - does a more expensive physiotherapist-delivered specific aquatic therapy program offer any additional benefits beyond that which can be achieved with a generic, community-based group aquatic exercise program? The provision of facilities and the additional costs associated with aquatic exercise when compared with home-based exercise programs will always be a factor that needs to be considered in an economic environment of ever-increasing pressure on health budgets. ${ }^{149}$ Clearly, more research is required to provide the evidence base to support the extensive expert opinion that considers aquatic intervention beneficial for people with OA of the hip or knee. 


\section{Disclosure}

The author reports no conflicts of interest in this work.

\section{References}

1. Anderson AS, Loeser RF. Why is osteoarthritis an age-related disease? Best Pract Res Clin Rheumatol. 2010;24:15-26.

2. Bosomworth NJ. Exercise and knee osteoarthritis: benefit or hazard? Can Fam Physician. 2009;55(9):871-878.

3. Friedrich MJ. Steps towards understanding, alleviating osteoarthritis will help aging population. JAMA. 1999;282(11):1023-1025.

4. Elliott B, Lloyd D, Ackland T. Biomechanical and neuromuscular considerations in the maintenance of an active lifestyle. In: Morris M, Schoo A, editors. Optimizing Exercise and Physical Activity in Older People. London: Butterworth-Heinemann; 2004.

5. Arden N, Nevitt MC. Osteoarthritis: epidemiology. Best Pract Res Clin Rheumatol. 2006;20(1):3-25.

6. Bennell KL, Hunt MA, Wrigley TW, Hunter DJ, Hinman RS. The effects of hip muscle strengthening on knee load, pain and function in people with knee osteoarthritis: a protocol for a randomised, single-blinded controlled trial. BMC Musculoskelet Disord. 2007;8:121-127.

7. Conaghan PG, Dickson J, Grant RL. Care and management of osteoarthritis in adults: summary of NICE guidance. BMJ. 2008; 336:502-503.

8. March L, Amatya B, Osborne RH, Brand C. Developing a minimum standard of care for treating people with osteoarthritis of the hip and knee. Best Pract Res Clin Rheumatol. 2010;24:121-145.

9. Fransen M. When is physiotherapy appropriate? Best Pract Res Clin Rheumatol. 2004;18(4):477-489.

10. Hurley MV. Muscle dysfunction and effective rehabilitation of knee osteoarthritis: what we know and what we need to find out. Arthritis Rheum. 2003;49(3):444-452.

11. Wang AW, Gilbey HJ, Ackland TR. Peri-operative exercise programs improve early return of ambulatory function after total hip arthroplasty. Am J Phys Med Rehabil. 2002;81(11):801-806.

12. Jones AC, Beaupre LA, Johnston DWC, Suarez-Almazor ME. Total joint arthroplasties: current concepts of patient outcomes after surgery [review]. Clin Geriatr Med. 2005;21:527-541.

13. Thomas A, Eichenberger G, Kempton C, et al. Recommendations for the treatment of knee osteoarthritis, using various therapy techniques, based on categorizations of a literature review. J Geriatr Phys Ther. 2009;32(1):33-38.

14. Zhang W, Moskowitz RW, Nuki G, et al. OARSI recommendations for the management of hip and knee osteoarthritis, part II: OARSI evidence-based, expert consensus guidelines. Osteoarthr Cartil. 2008;16:137-162.

15. Verhagen A, Bierma-Zeinstra SMA, Boers M, et al. Balneotherapy for osteoarthritis. Cochrane Database Syst Rev. 2007;(4):CD006864.

16. Schencking M, Otto A, Deutsch T, Sandholzer H. A comparison of Kneipp hydrotherapy with conventional physiotherapy in the treatment of osteoarthritis of the hip or knee: protocol of a prospective randomised controlled trial. BMC Musculoskelet Disord. 2009;10:104-113.

17. Fiscus KA, Kaminski TW, Powers ME. Changes in lower-leg blood flow during warm-, cold-, and contrast-water therapy. Arch Phys Med Rehabil. 2005;86:1404-1410.

18. Queneau P, Francon A, Graber-Duvernay B. Reflexions methodologiques sur 20 essais cliniques randomises en crenotherapie rhumatologique. Therapie. 2001;56:675-684.

19. WCPT. Education - Appendix A - Patient/Client Care/Management. http://www.wcpt.org/node/29560. Updated March 28, 2009. Accessed February 14, 2010.

20. Woollacott MH. Systems contributing to balance disorders in older adults [editorial]. J Gerontol A Biol Sci Med. 2000;55A(8):M424-M428.

21. Low Choy NL, Brauer S, Nitz JC. Age-related changes in strength and somatosensation during midlife. Ann NY Acad Sci. 2007;1114: $180-193$.
22. Morley JE. Sarcopenia revisited [editorial]. J Gerontol A Biol Sci Med. 2003;58A(10):909-910.

23. Topp R, Mikesky A, Thompson K. Determinants of four functional tasks among older adults: an exploratory regression analysis. J Orthop Sports Phys Ther. 1998;27(2):144-152.

24. Suetta C, Aagaard P, Magnusson SP, et al. Muscle size, neuromuscular activation, and rapid force characteristics in elderly men and women: effects of unilateral long-term disuse due to hip osteoarthritis. J Appl Physiol. 2007;102:942-948.

25. Rasch A, Bystrom AH, Dalen N, Berg HE. Reduced muscle radiological density, cross-sectional area, and strength of major hip and knee muscles in 22 patients with hip osteoarthritis. Acta Orthop. 2007;78(4):505-510.

26. Bennell KL, Hunt MA, Wrigley TW, Lim B-W, Hinman RS. Role of muscle in the genesis and management of knee osteoarthritis. Rheum Dis Clin North Am. 2008;34:731-754.

27. Hurley MV. Quadriceps weakness in osteoarthritis. Curr Opin Rheumatol. 1998;10:246-250.

28. Slemenda C, Brandt K, Heilman D, et al. Quadriceps weakness and osteoarthritis of the knee. Ann Intern Med. 1997;127: 97-104.

29. Winby CR, Lloyd DG, Besier TF, Kirk TB. Muscle and external load contribution to knee joint contact loads during normal gait. J Biomech. 2009;42:2294-2300.

30. Englund M. The role of biomechanics in the initiation and progression of osteoarthritis of the knee. Best Pract Res Clin Rheumatol. 2010;24:39-46.

31. Sims K. The development of hip osteoarthritis: implications for conservative management. Man Ther. 1999;4(3):127-135.

32. Childs JD, Sparto PJ, Fitzgerald GK, Bizzini M, Irrang JJ. Alterations in lower extremity movement and muscle activation patterns in individuals with knee osteoarthritis. Clin Biomech. 2004;19:44-49.

33. Yamada H, Koshino T, Sakai N, Saito T. Hip adductor muscle strength in patients with varus deformed knee. Clin Orthop Relat Res. 2001;386:179-185.

34. Lewek MD, Rudolph KS, Snyder-Mackler L. Control of frontal plane knee laxity during gait in patients with medial compartment knee osteoarthritis. Osteoarthr Cartil. 2004;12:745-751.

35. Sims KJ, Richardson CA, Brauer SG. Investigation of hip abductor activation in subjects with clinical unilateral hip osteoarthritis. Ann Rheum Dis. 2002;61(8):687-692.

36. Amaro A, Amando F, Duarte JA, Appell H-J. Gluteus medius muscle atrophy is related to contralateral and ipsilateral hip joint osteoarthritis. Int J Sports Med. 2007;28:1035-1039.

37. Ireland ML, Wilson JD, Ballantyne BT, Davis IM. Hip strength in females with and without patellofemoral pain. J Orthop Sports Phys Ther. 2003;33(11):671-676.

38. Mundermann A, Dyrby CO, Andriacchi TP. Secondary gait changes in patients with medial compartment knee osteoarthritis. Arthritis Rheum. 2005;52(9):2835-2844.

39. Chang A, Hayes K, Dunlop D, et al. Hip abduction moment and protection against medial tibiofemoral osteoarthritis progression. Arthritis Rheum. 2005;52(11):3515-3519.

40. Lord S, Rogers MW, Howland A, Fitzpatrick R. Lateral stability, sensori-motor function and falls in older people. J Am Geriatr Soc. 1999;47(9):1077-1081.

41. Johnson-Hilliard M, Martinez KM, Janssen I, et al. Lateral balance factors predict future falls in community-living older adults. Arch Phys Med Rehabil. 2008;89:1708-1713.

42. Sisto SA, Malanga GA. Osteoarthritis and therapeutic exercise. Am J Phys Med Rehabil. 2006;85:69-78.

43. Rasch A, Bystrom AH, Dalen N, Martinez-Carranza N, Berg HE. Persisting muscle atrophy two years after replacement of the hip. J Bone Joint Surg Br. 2009;91(B5):583-588.

44. Pelland L, Brosseau L, Wells G, et al. Efficacy of strength training exercises for osteoarthritis (part 1): a meta-analysis. Phys Ther Rev. 2004;9:77-108. 
45. Bennell K, Hinman RS. Exercise as a treatment for osteoarthritis. Curr Opin Rheumatol. 2005;17:634-640.

46. van Baar ME, Assendelft WJJ, Dekker J, Oostendorp RAB, Bijlsma JWJ. Effectiveness of exercise therapy in patients with osteoarthritis of the hip or knee: a systematic review of randomized clinical trials. Arthritis Rheum. 1999;42(7):1361-1369.

47. Fransen M, McConnell S. Exercise for osteoarthritis of the knee [review]. Cochrane Database Syst Rev. 2008;(4):CD004376.

48. Fransen M, McConnell S, Hernandez-Molina G, Reichenbach S. Exercise for osteoarthritis of the hip [review]. Cochrane Database Syst Rev. 2009;(3):CD007912.

49. Sharma L. Examination of exercise effects on knee osteoarthritis outcomes: why should the local mechanical environment be considered? Arthritis Rheum. 2003;49(2):255-260.

50. Hinman RS, Crossley KM. Patellofemoral joint osteoarthritis: an important subgroup of knee osteoarthritis [review]. Rheumatology. 2007;46:1057-1062.

51. Crossley KM, Vicenzino B, Pandy M, Schache AG, Hinman RS. Targeted physiotherapy for patellofemoral joint osteoarthritis: a protocol for a randomised, single-blind controlled trial. BMC Musculoskelet Disord. 2008;9:122-130.

52. Sims K. Masterclass. Assessment and treatment of hip osteoarthritis. Man Ther. 1999;4(3):136-144.

53. Bijlsma JWJ, Dekker J. A step forward for exercise in the management of osteoarthritis [editorial]. Rheumatology. 2005;44:5-6.

54. Becker BE. Aquatic therapy: scientific foundations and clinical rehabilitation applications. $P M \& R$. 2009;1:859-872.

55. Poyhonen T, Sipila S, Keskinen KL, Hautala A, Savolainen J, Malkia E. Effects of aquatic resistance training on neuromuscular performance in healthy women. Med Sci Sports Exerc. 2002;34(12): 2103-2109.

56. Johnson B, Stromme S, Adamczyk J, Tennoe K. Comparison of oxygen uptake and heart rate during exercise on land and in water. Phys Ther. 1977;57(3):273-278.

57. Butts NK, Tucker M, Smith R. Maximal responses to treadmill and deep water running in high school female cross country runners. Res Q Exerc Sport. 1991;62(2):236-239.

58. Poyhonen T, Keskinen K, Hautala A, Malkia E. Determination of hydrodynamic drag forces and drag coefficients on human leg/foot model during knee exercises. Clin Biomech. 2000;15: 256-260.

59. Goitz R, Towler M, Buschbacher L, Wilder R, Thacker J, Edlich RF. A new hydrofitness device for leg musculoskeletal conditioning. J Burn Care Rehabil. 1988;9(2):203-206.

60. Hillman MR, Matthews L, Pope JM. The resistance to motion through water of hydrotherapy table-tennis bats. Physiotherapy. 1987;73(10):570-572.

61. Geytenbeek J. Evidence for effective hydrotherapy. Physiotherapy. 2002;88(9):514-529.

62. Harrison R. A quantitative approach to strengthening exercises in the hydrotherapy pool. Physiotherapy. 1980;66(2):60.

63. Harrison RA, Allard LL. An attempt to quantify the resistances produced using the Bad Ragaz ring method. Physiotherapy. 1982;68(10):330-331.

64. Poyhonen T, Keskinen K, Kyrolainen H, Hautala A, Savolainen J, Malkia E. Neuromuscular function during therapeutic knee exercise under water and on dry land. Arch Phys Med Rehabil. 2001; 82:1446-1452.

65. Poyhonen T, Keskinen K, Hautala A, Savolainen J, Malkia E. Human isometric force production and electromyogram activity of knee extensor muscles in water and on dry land. Eur J Appl Physiol Occup Physiol. 1999;80(1):52-56.

66. Naemi R, Easson WJ, Sanders RH. Hydrodynamic glide efficiency in swimming. J Sci Med Sport. 2010; Jun 17 [Epub ahead of press].

67. Hartmann S, Huch R. Response of pregnancy leg edema to a single immersion exercise session. Acta Obstet Gynecol Scand. 2005;84:1150-1153.
68. Tidhar D, Drouin J, Shimony A. Aqua lymphatic therapy in managing lower extremity lymphedema. J Support Oncol. 2007;5(4): 179-183.

69. Rahmann A, Brauer S, Nitz JC. A specific inpatient aquatic physiotherapy program improves strength after total hip or knee replacement surgery: a randomized controlled trial. Arch Phys Med Rehabil. 2009;90:745-755.

70. Becker BE. Biophysiologic Aspects of Hydrotherapy. In: Cole AJ, Becker BE, editors. Comprehensive Aquatic Therapy. Philadelphia: Butterworth Heinemann; 2004.

71. Vaile J, Halson S, Gill N, Dawson B. Effect of hydrotherapy on the signs and symptoms of delayed onset muscle soreness. Eur J Appl Physiol. 2008; 102:447-455.

72. Phillips VK, Legge M, Jones LM. Maximal physiological responses between aquatic and land exercise in overweight women. Med Sci Sports Exerc. 2008;40(5):959-964.

73. Sheldahl LM, Wann LS, Clifford PS, Tristani FE, Wolf LG, Kalbfleisch JH. Effect of central hypervolemia on cardiac performance during exercise. J Appl Physiol. 1984;57(6):1662-1667.

74. Lund $\mathrm{H}$, Weile $\mathrm{U}$, Christensen $\mathrm{R}$, et al. A randomized controlled trial of aquatic and land-based exercise in patients with knee osteoarthritis. J Rehabil Med. 2008;40:137-144.

75. Dalbeth N, Arroll B. Commentary: controversies in NICE guidance on osteoarthritis. BMJ. 2008;336:504-505.

76. Bartels EM, Lund H, Hagen KB, Dagfinrud H, Christensen R, Danneskiold-Samsoe B. Aquatic exercise for the treatment of knee and hip osteoarthritis [review]. Cochrane Database Syst Rev. 2007;(4): CD005523.

77. Petrick MA, Paulsen T, George J. Comparison between quadriceps muscle strengthening on land and in water. Physiotherapy. 2001;87(6):310-317.

78. Suomi R, Lindauer S. Effectiveness of Arthritis Foundation Aquatic Program on strength and range of motion in women with arthritis. J Aging Phys Act. 1997;5:341-351.

79. Wang T-J, Belza BL, Thompson E, Whitney JD, Bennett K. Effects of aquatic exercise on flexibility, strength and aerobic fitness in adults with osteoarthritis of the hip or knee. JAdv Nurs. 2007; 57(2): 141-152.

80. Alexander MJL, Butcher JE, MacDonald PB. Effect of a water exercise program on walking gait, flexibility, strength, self-reported disability and other psycho-social measures of older individuals with arthritis. Physiother Can. 2001;53(3):203-211.

81. Cochrane T, Davey RC, Matthes Edwards SM. Randomised controlled trial of the cost-effectiveness of water-based therapy for lower limb osteoarthritis. Health Technol Assess. 2005;9(31):1-130.

82. Lin S-C, Davey R, Cochrane T. Community rehabilitation for older adults with osteoarthritis of the lower limb: a controlled clinical trial. Clin Rehabil. 2004;18:92-101.

83. Hinman RS, Heywood SE, Day AR. Aquatic physiotherapy for hip and knee osteoarthritis: results of a single-blind randomised controlled trial. Phys Ther. 2007;87(1):32-43.

84. Dziedzic K, Jordan JL, Foster NE. Land- and water-based exercise therapies for musculoskeletal conditions. Best Pract Res Clin Rheumatol. 2008;22(3):407-418.

85. Hernandez-Molina G, Reichenbach S, Zhang B, Lavalley M, Felson D. Effect of therapeutic exercise for hip osteoarthritis pain: results of a meta-analysis. Arthritis Rheum. 2008;59(9):1221-1228.

86. Green J, McKenna F, Redfern EJ, Chamberlain MA. Home exercises are as effective as outpatient hydrotherapy for osteoarthritis of the hip. Br J Rheumatol. 1993;32:812-815.

87. Wyatt FB, Milam S, Manske RC, Deere R. The effects of aquatic and traditional exercise programs on persons with knee osteoarthritis. J Strength Cond Res. 2001;15(3):337-340.

88. Silva LE, Valim V, Pessanha AP, et al. Hydrotherapy versus conventional land-based exercise for the management of patients with osteoarthritis of the knee: a randomized clinical trial. Phys Ther. 2008;88(1): $12-21$. 
89. Gill SD, McBurney H, Schulz DL. Land-based versus pool-based exercise for people awaiting joint replacement surgery of the hip or knee: results of a randomised controlled trial. Arch Phys Med Rehabil. 2009;90:388-394.

90. Foley A, Halbert J, Hewitt T, Crotty M. Does hydrotherapy improve strength and physical function in patients with osteoarthritis - a randomised controlled trial comparing a gym based and a hydrotherapy strengthening programme. Ann Rheum Dis. 2003;62:1162-1167.

91. Fransen M, Nairn L, Winstanley J, Lam P, Edmonds J. Physical activity for osteoarthritis management: a randomized controlled clinical trial evaluating hydrotherapy or Tai Chi classes. Arthritis Rheum. 2007;57(3):407-414.

92. Jordan KM, Arden NK, Doherty M, et al. EULAR recommendations 2003: an evidence based approach to the management of knee osteoarthritis: report of a task force of the Standing Committee for International Clinical Studies including therapeutic trials (ESCISIT). Ann Rheum Dis. 2003;62:1145-1155.

93. Nicholas MK. Pain management in musculoskeletal conditions. Best Pract Res Clin Rheumatol. 2008;22(3):451-470.

94. Melzac R. Pain and the neuromatrix in the brain. J Dent Educ. 2001;65(12):1378-1382.

95. Crowninshield RD, Rosenberg AG, Sporer SM. Changing demographics of patients with total joint replacement. Clin Orthop Relat Res. 2006;443:266-272.

96. Kidd BL. Osteoarthritis and joint pain. Pain. 2006;123:6-9.

97. Hurley MV, Newham DJ. The influence of arthrogenous muscle inhibition on quadriceps rehabilitation of patients with early, unilateral osteoarthritic knees. Br J Rheumatol. 1993;32:127-131.

98. Hunt MA, Birmingham TB, Bryant D, et al. Lateral trunk lean explains variation in dynamic knee joint load in patients with medial compartment knee osteoarthritis. Osteoarthr Cartil. 2008;16: 591-599.

99. Manetta J, Franz LH, Moon C, Perell KL, Fang M. Comparison of hip and knee muscle moments in subjects with and without knee pain. Gait Posture. 2002;16:249-254.

100. Hinman RS, Bennell KL, Metcalf BR, Crossley K. Balance impairment in individuals with symptomatic knee osteoarthritis: a comparison with matched controls using clinical tests. Rheumatology. 2002;41:1388-1394.

101. Lu TW, Chen HL, Wang TM. Obstacle crossing in older adults with medial compartment knee osteoarthritis. Gait Posture. 2007;26:553-559.

102. Pandya NK, Piotrowski GA, Pottenger L, Draganich LF. Pain relief in knee osteoarthritis reduces the propensity to trip on obstacles. Gait Posture. 2007;25:106-111.

103. Hassan BS, Doherty SA, Mockett S, Doherty M. Effect of pain reduction on postural sway, proprioception and quadriceps strength in subjects with knee osteoarthritis. Ann Rheum Dis. 2002;61: $422-428$

104. Hurwitz DE, Sharma L, Andriacchi TP. Effect of knee pain on joint loading in patients with osteoarthritis. Curr Opin Rheumatol. 1999;11:422-426.

105. Van Baar ME, Dekker J, Oostendorp RAB, Bijl D, Lemmens JAM, Bijlsma JWJ. The effectiveness of exercise therapy in patients with osteoarthritis of the hip or knee: a randomised clinical trial. J Rheumatol. 1998;25:2432-2439.

106. Gerber LH. Exercise and arthritis. Bull Rheum Dis. 1990;39(6):1-9.

107. Borjesson M, Robertson E, Weidenhielm L, Mattsson E, Olsson E. Physiotherapy in knee osteoarthrosis: effect on pain and walking. Physiother Res Int. 1996;1(2):89-97.

108. Bellamy N. The WOMAC knee and hip osteoarthritis indices: development, validation, globilization and influences on the development of the AUSCAN hand osteoarthritis indices. Clin Exp Rheumatol. 2005;23(5 Suppl 39):S148-S153.

109. Stratford PW, Kennedy DM, Riddle DL. New study design evaluated the validity of measures to assess change after hip or knee arthroplasty. J Clin Epidemiol. 2009;62:347-352.
110. Podsiadlo D, Richardson S. The Timed "Up \& Go": a test of basic functional mobility for frail elderly persons. J Am Geriatr Soc. 1991;39:142-148.

111. Fransen M, Crosbie J, Edmunds J. Reliability of gait measurements in people with osteoarthritis of the knee. Phys Ther. 1997;77(9):944-953.

112. Stratford PW, Kennedy DM, Woodhouse LJ. Performance measures provide assessments of pain and function in people with advanced osteoarthritis of the hip or knee. Phys Ther. 2006; 86(11):1489-1496.

113. Brandt KD. The importance of nonpharmacological approaches in management of osteoarthritis. Am J Med. 1998;105(1B):S39-S44.

114. Jamtvedt G, Thuve Dahm K, Christie A, et al. Physical therapy interventions for patients with osteoarthritis of the knee: An overview of systematic reviews. Phys Ther. 2008;88(1):123-136.

115. Lange AK, Vanwanseele B, Fiatarone Singh MA. Strength training for treatment of osteoarthritis of the knee: a systematic review. Arthritis Rheum. 2008;59(10):1488-1494.

116. Moe R, Haavardsholm EA, Christie A, Jamtvedt G, Thuve Dahm K, Birger Hagen K. Effectiveness of nonpharmacological and nonsurgical interventions for hip osteoarthritis: an umbrella review of high-quality systematic reviews. Phys Ther. 2007;87(12):1716-1727.

117. Arokoski JPA. Physical therapy and rehabilitation programs in the management of hip osteoarthritis. Eura Medicophys. 2005;41:155-161.

118. Wilder FV, Barrett JP, Farina EJ. Exercise and osteoarthritis: are we stopping too early? Findings from the Clearwater Exercise Study. J Aging Phys Act. 2006;14:169-180.

119. Deyle GD, Henderson NE, Matekel RL, Ryder MG, Garber MB, Allison SC. Effectiveness of manual physical therapy and exercise in oseoarthritis of the knee. Ann Intern Med. 2000;132(3):173-181.

120. Deyle GD, Allison SC, Matekel RL, et al. Physical therapy treatment effectiveness for osteoarthritis of the knee: A randomized comparison of supervised clinical exercise and manual therapy procedures versus a home exercise program. Phys Ther. 2005;85(12):1301-1317.

121. Zhang W, Moskowitz RW, Nuki G, et al. OARSI recommendations for the management of hip and knee osteoarthritis, part 1: critical appraisal of existing treatment guidelines and systematic review of current research evidence. Osteoarthr Cartil. 2007;15:981-1000.

122. Hall J, Swinkels A, Briddon J, McCabe C. Does aquatic exercise relieve pain in adults with neurologic or musculoskeletal disease? A systematic review and meta-analysis of randomized controlled trials. Arch Phys Med Rehabil. 2008;89:873-883.

123. Rahmann A. A comparison between ward and aquatic exercise for acute orthopaedic inpatients. In: 8th International Physiotherapy Conference; 2004; Adelaide, Australia.

124. Prins J, Cutner D. Aquatic therapy in the rehabilitation of athletic injuries. Clin Sports Med. 1999;18(2):447-461.

125. Harrison RA, Hillman M, Bulstrode S. Loading of the lower limb when walking partially immersed: implications for clinical practice. Physiotherapy. 1992;78(3):164-166.

126. Becker BE, Garrett G. Hydrotherapuetic applications in arthritis rehabilitation. In: Cole AJ, Becker BE, editors. Comprehensive Aquatic Therapy. Philadelphia: Butterworth-Heinemann; 2004: 207-226.

127. Camilotti BM, Rodacki ALF, Israel VL, Fowler NE. Stature recovery after sitting on land and in water. Man Ther. 2009;14:685-689.

128. Lee C. Orthopaedics. In: Reid Campion M, editor. Hydrotherapy: Principles and Practice. Oxford: Butterworth-Heinemann; 1997.

129. Tinsley LM. Rheumatic diseases. In: Reid Campion M, editor. Hydrotherapy: Principles and Practice. Oxford: Butterworth-Heinemann; 1997.

130. Westby MD. A health professional's guide to exercise prescription for people with arthritis: a review of aerobic fitness activities. Arthritis Care Res. 2001;45:501-511.

131. Altman RD, Hochberg MC, Moskowitz RW, Schnitzer TJ. Recommendations for the medical management of osteoarthritis of the hip or knee: 2000 update. Arthritis Rheum. 2000;43(9):1905-1915. 
132. Brosseau L, Pelland L, Wells G, et al. Efficacy of aerobic exercises for osteoarthritis (part 2): a meta-analysis. Phys Ther Rev. 2004;9:125-145.

133. Brosseau L, MacLeay L, Robinson VA, Tugwell P, Wells G. Intensity of exercise for the treatment of osteoarthritis. Cochrane Database Syst Rev. 2003;(2):CD004259.

134. Mangione KK, McCully K, Gloviak A, Lefebvre I, Hofmann M, Craik R. The effects of high-intensity and low-intensity cycle ergometry in older adults with knee osteoarthritis. J Gerontol. 1999;54A(4):M184-M190.

135. Minor MA, Hewett JE, Webel RR, Anderson SK, Kay DR. Efficacy of physical conditioning exercise in patients with rheumatoid arthritis and osteoarthritis. Arthritis Rheum. 1989;32(11):1396-1405.

136. Kovar P, Allegrante JP, MacKenzie CR, Petersen MGE, Gutin B, Charlson ME. Supervised fitness walking in patients with osteoarthritis of the knee. Ann Intern Med. 1992;116:529-534.

137. Peloquin L, Bravo G, Gauthier P, Lacombe G, Billiard JS. Effects of a cross-training exercise program in persons with osteoarthritis of the knee: a randomised controlled trial. J Clin Rheumatol. 1999;5(3):126-136.

138. Chevutschi A, Alberty M, Lensel G, Pardessus V, Thevenon A. Comparison of maximal and spontaneous speeds during walking on dry land and water. Gait Posture. 2009;29:403-407.

139. Masumoto K, Shono T, Hotta N, Fujishima K. Muscle activation, cardiorespiratory response, and rate of perceived exertion in older subjects while walking in water and on dry land. $J$ Electromyogr Kinesiol. 2008;18:581-590.

140. Hall J, Grant J, Blake D, Taylor G, Garbutt G. Cardiorespiratory responses to aquatic treadmill walking in patients with rheumatoid arthritis. Physiother Res Int. 2004;9(2):59-73.
141. Roesler H, Haupenthal A, Schutz GR, de Souza PV. Dynamometric analysis of the maximum force applied in aquatic human gait at $1.3 \mathrm{~m}$ of immersion. Gait Posture. 2006;24:412-417.

142. Miyoshi T, Shirota T, Yamamoto S-I, Nakazawa K, Akai M. Functional roles of lower-limb joint moments while walking in water. Clin Biomech. 2005;20:194-201.

143. Chaloupka EC, Kang J, Mastranglo MA, Donnelly MS. Cardiorespiratory and metabolic responses during forward and backward walking. J Orthop Sports Phys Ther. 1997;25(5):302-306.

144. Hamer P, Morton A. Water-running: training effects and specificity of aerobic, anaerobic and muscular parameters following an eight-week interval training programme. Aust J Sci Med Sport. 1990;22(1):13-22.

145. Takeshima N, Rogers ME, Watanabe E, et al. Water-based exercise improves health-related aspects of fitness in older women. Med $\mathrm{Sci}$ Sports and Exer. 2002;34(3):544-551.

146. Jackson A. Hydrotherapy as experienced by outpatients in a general hospital. Brit J Ther Rehabil. 1996;3(11):601-608.

147. Gyurcsik NC, Estabrooks PA, Frahm-Templar MJ. Exercise-related goals and self-efficacy as correlates of aquatic exercise in individuals with arthritis. Arthritis Rheum. 2003;49(3):306-313.

148. Geytenbeek J. Aquatic physiotherapy evidence-based practice guide. Systematic review. National Aquatic Physiotherapy Group and Australian Physiotherapy Association; 2008. http://www. physiotherapy.asn.au/index.php/groups/aquatic-physiotherapy/ resources-clinical. Accessed Mar 10, 2010.

149. Patrick DL, Ramsey SD, Spencer AC, Kinne S, Belza BL, Topolski TD. Economic evaluation of aquatic exercise for persons with osteoarthritis. Med Care. 2001;39(5):413-424.
Open Access Journal of Sports Medicine

\section{Publish your work in this journal}

Open Access Journal of Sports Medicine is an international, peer-reviewed, open access journal publishing original research, reports, reviews and commentaries on all areas of sports medicine. The manuscript management system is completely online and includes a very quick and fair peer-review system.

\section{Dovepress}

Visit http://www.dovepress.com/testimonials.php to read real quotes from published authors. 